\title{
Rechtsgeschichte
}

\section{Thomas Kupka}

Averroes als Rechtsgelehrter 


\section{Averroes als Rechtsgelehrter*}

Ibn Rušd (II26-II98) ist unter dem lateinischen Namen Averroes in der westlichen Philosophie vor allem als Aristoteleskommentator und Protagonist des Aristotelismus im mittelalterlichen Universalienstreit bekannt. Er gilt heute als einer der wichtigsten Vertreter eines islamischen Rationalismus, wenngleich er zu seiner Zeit in der muslimischen Welt weitgehend abgelehnt wurde. Viele arabische Intellektuelle und die Mehrzahl der europäischen Orientalisten setzen mit seinem Tode das Ende der Philosophie im Islam überhaupt an. Erst im zwanzigsten Jahrhundert beginnt dies langsam aufzubrechen und man versucht, Averroes als einen der bedeutendsten islamischen Denker zurückzugewinnen. ${ }^{\mathrm{I}}$

Auch die von Franz Schupp herausgegebene und kommentierte Schrift Die entscheidende Abhandlung und die Urteilsfällung über das Verhältnis von Gesetz und Philosophie, wird zur Rezeption gewiss ihren Beitrag leisten. Gleichzeitig gibt sie Gelegenheit, den Blick auf den »lateinischen Averroismus ", der sich erkenntnistheoretisch mit Aristoteles von der neuplatonischen Universalienlehre verabschiedete und später von Wilhelm von Ockham gar zur Entwicklung einer nominalistischen Weltsicht beigezogen wurde, um die Frage zu ergänzen, wie Averroes das Verhältnis der Philosophie zum islamischen Recht und den dortigen »erkenntnistheoretischen " Voraussetzungen bestimmte. Hier gibt es in Teilen durchaus Widersprüchliches zu konstatieren. So vertritt er im Universalienstreit, dass die Erkenntnis mit der sinnlichen Wahrnehmung des Einzeldings beginnt, worauf das Bewahren des Eindrucks durch die Vorstellungskraft folgt und schließlich das den Einzeldingen Allgemeine durch Wiederholung des Eindrucks mittels »Gedächtniskraft " erkannt wird. Diese Erkenntnisfolge zu bewerkstelligen ist Aufgabe des »tätigen Intellekts «, der aus den individuellen Bildern die Universalien oder Intelligibilia abstrabiert. Damit gibt Averroes die Idee von einem außerweltlichen Dator formarum (wāhib al-şūwar) auf und zugleich die bis dahin vorherrschende Emanationslehre Avicennas (980-I037). Die averroistische Abstraktion, wie Alain de Libera pointiert, ist nicht Donation, sondern Reduktion. Und sie ist nicht schlichte Entmaterialisierung, sondern sukzessive Universalisierung. ${ }^{2}$

Fürs islamische Recht hingegen ist Averroes ganz anderer Meinung. Hier ist Gott Anfang wie Ende von allem und der Koran das als Offenbarung aufgefasste Gesetz. Die Geltung des islamischen Gesetzes wird vorausgesetzt und steht nicht zur Diskussion, auch nicht für Philosophen. Averroes kennt hier weder Willensfreiheit noch selbständige Urteilsfindung. Vom Intellectus agens keine Spur mehr. Allerdings ist die geistliche Elite durchaus aufgefordert, über die rechte Interpretation des Gesetzes zu philosophieren. Dem Volk ist das jedoch unbedingt vorzuenthalten, und wer dagegen verstößt ist zu bestrafen, was zumindest an dieser Stelle ganz gegen Aristoteles steht, der ja gleich im ersten Satz seiner Metaphysik bestimmt, dass alle Menschen von Natur aus nach Wissen streben. Für Léon Gauthier, der 1942 die erste französische Übersetzung der Entscheidenden Abhandlung besorgte, ist Averroes denn auch »Rationalist, soweit er sich an die Philosophen wendet [...]. Für sie gibt es weder Mysterien noch sogenannte Wunder. " Er ist hingegen "antirationalistisch, fideistisch, wenn es sich um das Volk handelt $«{ }^{3}$
* Averroes, Die entscheidende Abhandlung und die Urteilsfällung über das Verhältnis von Gesetz und Philosophie, arabisch-deutsch, mit einer Einleitung und kommentierenden Anmerkungen übersetzt von Franz Schupp, Hamburg: Felix Meiner 2009, cxxx, 338 S., ISBN 978-3-7873-I 884-I

స్ํำ

Kupka, Averroes als Rechtsgelehrter
I Ausführlich ANKe von KüGELGEN, Averroes und die arabische Moderne, Leiden I994.

2 Alain de Libera, Der Universalienstreit, München 2005, 2 I 7. Eine ganz interessante Idee hat BARRY S. Kogan, Averroës and the Theory of Emanation, in: Mediaeval Studies 43 (I98I) 384404, 395 ff., 399, der eine Art umgekehrte (also auf- statt absteigende) Emanation in Averroes'
Kausalitätslehre ausmacht. Zur o.g. Ablehnung eines außerweltlichen Formengebers siehe ebd., $40 \mathrm{If}$.

3 LÉon Gauthier, La théorie d'Ibn Rochd (Averroès) sur les rapports de la religion et de la philosophie, Paris I909, I80 - zitiert nach KüGELGEN (Fn. I), I 4 . 
Indes war das für die Zeit nicht ungewöhnlich und keineswegs nur ein Problem des Islam. Auch in der lateinischen Scholastik hatten es Philosophie und Wissenschaft bekanntlich schwer. Man denke nur an die Replatonisierung des Peripatismus durch Albertus Magnus, der die aristotelischen Schriften via Averroes rezipierte, oder an die Instrumentalisierung der aristotelischen Politiklehre zur Befestigung der päpstlichen Vorherrschaft bei Thomas von Aquin. Leo Strauss hat ja ganz richtig gesehen, »wie die mittelalterlichen Philosophen die Religion verstehen: sie verstehen sie nicht als >Geltungsgebiet‘, auch nicht als eine >Richtung des Bewusstseins`, am allerwenigsten als ein ’Kulturgebiet`, sondern als Gesetz «. ${ }^{4}$ Das führt auch bei ihm zur Frage nach dem Verhältnis von Philosophie und Gesetz, wobei er mit dem »offenbarungsgläubigen Rationalismus « der mittelalterlichen und vor allem jüdischen Rationalisten in der Folge Maimonides', die er mit der Entscheidenden Abhandlung des Averroes konfrontierte, ${ }^{5}$ zumindest geliebäugelt hat. Jedenfalls hat seit seiner Spinoza-Schrift auch Strauss ein »philosophisches Leben « nur der Elite vorbehalten, während die Menge, in ausdrücklicher Rückorientierung auf die vormoderne politische Theorie, den Halt der Religion benötige, damit verlässliche Ordnung gewährleistet ist: »Die Religion stellt sich [...] dar als eine Kombination aus Elementen der Vernunft und des Aberglaubens: der Vernunft entstammt ihr Ziel, dem Aberglauben ihr Mittel «. ${ }^{6}$

An Averroes' Entscheidender Abhandlung lässt sich nachvollziehen, welche Folgen das haben kann. Sie beginnt mit der Frage, "ob die Überlegung über die Philosophie und die Wissenschaften der Logik vom Gesetz erlaubt, verboten oder angeordnet ist, und zwar entweder als Empfehlung oder als Verpflichtung « $(\mathbb{S} I)$. Ge- meint ist, wie gesagt, das islamische Gesetz (d. h. der Koran und die Überlieferung), und Averroes beantwortet die Frage sogleich damit, dass die »Vernunftüberlegung über die existierenden Dinge « selbstverständlich verpflichtend ist, soweit sie nur, und dies wieder ganz gegen Aristoteles, "nicht mehr ist « als der »Hinweis « auf Gott als den »Hersteller « der Welt (\$2). Damit sind Philosophie und Wissenschaft auf die Erkenntnis Gottes und die durch ihn veranstaltete Welt beschränkt. Alles andere ist »unzulässige Neuerung « $\left(b^{\prime} d^{\prime} a\right)$ oder »Unglaube « $(k u f r)$. Und während die »unzulässige Neuerung « sich auf eine Überzeugung oder Praxis bezieht, die nicht auf die Zeit des Propheten zurückgeht, weshalb Averroes sich genötigt sieht, die Beschäftigung des Philosophierens gegen den möglichen Vorwurf der unzulässigen Neuerung zu verteidigen ( $\$ 4 \mathrm{III})$, liegt Unglaube auch für ihn bereits vor, wenn die in $\mathbb{} 2$ gesetzten Grenzen des Philosophierens überschritten werden. Damit ist Philosophie im Wesentlichen Interpretation. Das ist sie heute zwar auch noch, nur darf sie sich den zu interpretierenden Gegenstand seit der Aufklärung zumindest selbst aussuchen. Der averroistische Islam hingegen verbleibt in einem Begründungszirkel, der wohl für jede Schriftreligion charakteristisch ist: Gott hat im offenbarten Gesetz die »wahre « Interpretation implizit gleich mit verkündet und »der Beweis [für die richtige Interpretation] liegt nur dann vor, wenn er zur Wahrheit führt« (\$I6 III).

Irren allerdings ist durchaus erlaubt - aber nur für die »Gelehrten [...], wenn sie die schwierigen Dinge überlegen « $(\mathbb{S} 23 \mathrm{II})$. Für alle anderen ist es Sünde, da Unglaube ( $\mathbb{S} 24 \mathrm{ff}$., $33 \mathrm{f}$.) womit wir wieder bei Leo Strauss sind. Da wundert es ein wenig, dass Strauss sich überhaupt fragt, ob man »die durch das Gesetz ermächtigte Philosophie frei« nennen kann, die

4 Leo Strauss, Philosophie und Gesetz (1935), in: Gesammelte Schriften (ed. Heinrich Meier), Bd. II, Stuttgart I997, 3-I23, 47. 5 Strauss (Fn. 4), bes. $69 \mathrm{ff}$.

6 STRauss, Die Religionskritik Spinozas (I930), in: Gesammelte Schriften (Fn. 4), Bd. I (2. Aufl. 200I), I-330, 3 IO. 
Averroes hier im Sinn hat. ${ }^{7}$ Mehr noch aber befremdet sein Ergebnis: »[I]hre Freiheit beruht auf ihrer [religiösen] Bindung. « Und als müsste er sich davon erst selbst überzeugen, stellt er abschließend fest, »dass Ibn Ruschd nicht etwa der Voltaire des zwölften Jahrhunderts " war. ${ }^{8}$

Das sieht natürlich auch Franz Schupp so, wenngleich aus anderer Perspektive. In der Einleitung stellt er lieber das »rationale Minimum « heraus, das er in Averroes entdeckt (LXVIIf., LXXII, CV). An manchen Stellen mag selbst das ein wenig zu wohlwollend ausfallen, wenn auch mit dem richtigen Ziel, die interkulturelle Rezeption und damit Öffnung des Islams zu fördern. Doch lässt Schupp keinen Zweifel daran, dass die Dichotomie von religiöser Elite und Masse, die die Aufklärung des Volkes verbietet und die Verheimlichung der wahren Überzeugungen (taqiyya) und Absichten sowie gar bewusste Fehlleitungen (etwa betreffend Himmel und Hölle) erlaubt $(\mathbb{S} \mathbb{S} 43,45,47$; XC), »für den modernen Leser " schwer erträglich ist (S. I6I und XCVIII f.). Daran zu erinnern ist vielleicht

\section{Schattenboxen*}

Die Untersuchung des an der Universität von Washington (Tacoma) lehrenden Politikwissenschaftlers Turan Kayaoğlu befasst sich trotz anderslautendem Titel nicht mit Exterritorialität, sondern mit den Bedingungen, die zu ihrer Abschaffung geführt haben. An den Beispielen Japan, Osmanisches Reich und China analysiert der Autor den Reformprozess im I9. und 20. Jahrhundert im Bereich der Justiz und Rechtskodifizierung, welcher nach dem Dafürhalten der Großmächte Großbritannien und nicht unangemessen bei allem Verständnis, das man im Westen für den Islam aufzubringen versucht, und angesichts dessen, dass Averroes im arabischen Raum heute als Leitfigur eines islamischen Rationalismus gehandelt wird.

Bleibt noch ein Wort zur Ausstattung des Buches. Neben der sorgfältigen Einleitung, die dem Leser einen schnellen Zugriff ermöglicht und über die angestellten Erwägungen zur Übersetzung aufklärt, enthält der Band eine ausführliche Kommentierung, die sich über Fußnoten im deutschen Textteil erschließt. Im Anhang befinden sich darüber hinaus einzelne Artikel zu wichtigen Zeitgenossen Averroes' und zu themenrelevanten Sachbegriffen. Ein Literaturverzeichnis, ein Verzeichnis der von Averroes verwendeten Koranzitate, ein ausführliches arabisches Wörterverzeichnis und ein Namens- und Sachregister vervollständigen den Band, der mithin philologisch keine Wünsche offen lässt.

\section{Thomas Kupka}

Amerika die Voraussetzung schuf, exterritoriale
Gerichtsbarkeit abzuschaffen und die vormals
dominierten Staaten als gleichwertige Mitglieder
der Völkerrechtsfamilie anzuerkennen. Dabei
sind die drei Beispiele gut gewählt, ist das Zeit-
alter der Exterritorialität doch bis heute fester
Bestandteil im außenpolitischen Diskurs der be-
troffenen Staaten geblieben (46).
Ohne unmittelbare koloniale Kontrolle
über asiatische und afrikanische Überseegebiete
auszuüben, diente exterritoriale Gerichtsbarkeit
7 Strauss (Fn. 4) 7 I.

8 Strauss (Fn. 4) $74 \mathrm{f}$.

\footnotetext{
* Turan KayaoĞLu, Legal Imperialism. Sovereignty and Extraterritoriality in Japan, the Ottoman Empire, and China, New York: Cambridge University Press 20I0, VIII, 237 p., ISBN 978-0-52I-7659I-6
} 